\title{
PRECISION AND ACCURACY PARAMETERS IN STRUCTURED LIGHT 3-D SCANNING
}

\author{
Eypór R. Eiríksson ${ }^{\mathrm{a}}$, Jakob Wilm ${ }^{\mathrm{a}}$, David B. Pedersen ${ }^{\mathrm{b}}$ and Henrik Aanæs ${ }^{\mathrm{a}}$ \\ ${ }^{a}$ Department of Applied Mathematics and Computer Science \\ ${ }^{\mathrm{b}}$ Department of Mechanical Engineering \\ Technical University of Denmark \\ \{eruei,jakw, aanes\}@dtu.dk, dbpe@mek.dtu.dk
}

KEY WORDS: Structured Light, 3d Scanning, Accuracy Assessment, VDI 2634 (2)

\begin{abstract}
:
Structured light systems are popular in part because they can be constructed from off-the-shelf low cost components. In this paper we quantitatively show how common design parameters affect precision and accuracy in such systems, supplying a much needed guide for practitioners. Our quantitative measure is the established VDI/VDE 2634 (Part 2) guideline using precision made calibration artifacts. Experiments are performed on our own structured light setup, consisting of two cameras and a projector. We place our focus on the influence of calibration design parameters, the calibration procedure and encoding strategy and present our findings. Finally, we compare our setup to a state of the art metrology grade commercial scanner. Our results show that comparable, and in some cases better, results can be obtained using the parameter settings determined in this study.
\end{abstract}

\section{INTRODUCTION}

Structured Light (SL) systems enable robust high quality capture of 3D geometry, and are actively used throughout several fields. These systems can be constructed using commercial off the shelf (COTS) hardware, making them accessible and affordable. The obtainable accuracy and precision of such systems vary considerably, and are mainly functions of several design parameters. The influence of these parameters has not been studied extensively in the literature. Previously, no combined study has systematically investigated the effect of common parameter choices on the final result and quantified them using an established standard.

To address the lack of work in this regard, we investigate how common design choices influence precision and accuracy. Our analysis is based on our own active stereo-vision setup consisting of two industrial cameras and a consumer projector. We empirically show our parameter selection such that maximum performance is obtained, and quantify using the VDI/VDI 2634 (Part 2) guideline. Finally, we compare our results to a commercial metrology grade scanner (GOM ATOS III Triple Scan) as a benchmark against state of the art, with decent results. Throughout this study we seek to employ widely available and accepted methods $\&$ models used in such systems to obtain easily reproducible results.

The contribution of this paper lies in the attempt to quantitatively answer the following questions

- What calibration parameters should be included in the calibration procedure?

- What angular range of observations is required in the calibration procedure?

- How many observations are required for calibration?

- Which SL encoding strategy is the overall best performer?

We believe this to be valuable information for practitioners wanting to build their own system, e.g. as part of research projects or industrial implementations.
This paper is structured as follows. Section 2. covers related work. Section 3. gives an overview of our experimental setup. Section 4., 5. and 6. covers our investigations on calibration parameters, calibration observations and encoding strategies respectively. In section 7. we compare our system to a commercial system and finally, we conclude in section 8 .

\section{RELATED WORK}

Much work has been devoted to the field of SL systems e.g. (24, $7,6,11,30)$. These contributions have mostly dealt with the methodological development of such systems whereas less focus has been placed on quantitative accuracy and precision analysis. One of the most important factors with respect to accuracy is system calibration. While recent focus has been placed on projectorcamera calibration $(32,18,17)$, we here consider an active stereo vision setup $(14,31,33)$, without projector calibration. Precision is considered to be mostly dependent on the encoding strategy. A vast selection of methods have been proposed, see $(26,10,8)$ for recent surveys. While many of these methods aim to reduce the number of patterns, the amount of outliers and computational complexity, less focus has been placed on precision. Here, we compare selected encoding strategies from a precision and accuracy perspective.

Characterising SL systems in terms of accuracy is a challenging and ongoing problem, which despite its relevance has only seen few published guidelines and standards. The only currently published standard is the German VDI/VDE 2634 Part 2 guideline (1, 13), Optical 3-D measuring systems - Optical systems based on area scanning. This guideline aims to capture the complex nature of such a system, using a number of length and shape measurements throughout the scanning volume. Researchers have already accepted this guideline for evaluation of 3D scanning systems (4, $20,3,2)$. We here argue that the guideline is lacking to some extent. Firstly, it fails to capture frequency response characteristics of SL systems using the proposed low frequency artifacts. Lastly, the artifacts are optically ideal for SL scanning. Therefore, results only indicate 'best case' results, given that particular material. The standard is however well suited for relative measures $e . g$. for acceptance testing and benchmarking purposes. 


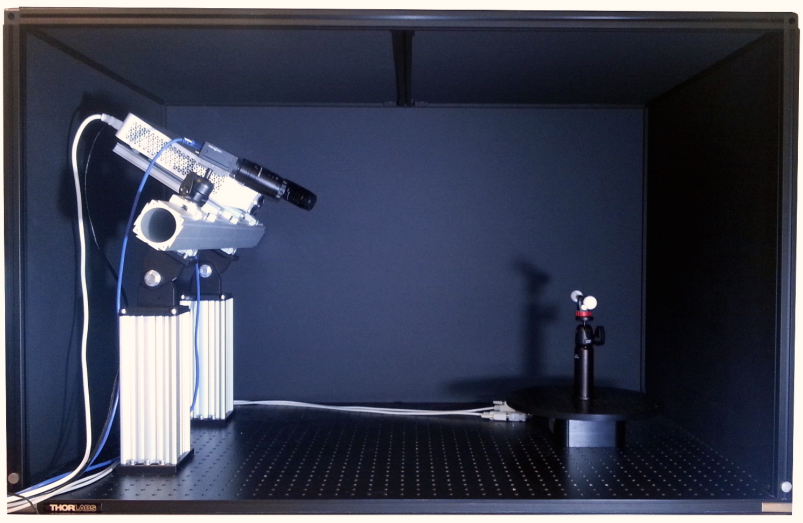

Figure 1: Our structured light system setup with two highresolution industrial cameras, a Full HD LED projector and a rotation stage mounted on a rigid aluminum mount. Specifications are given in Table 1.
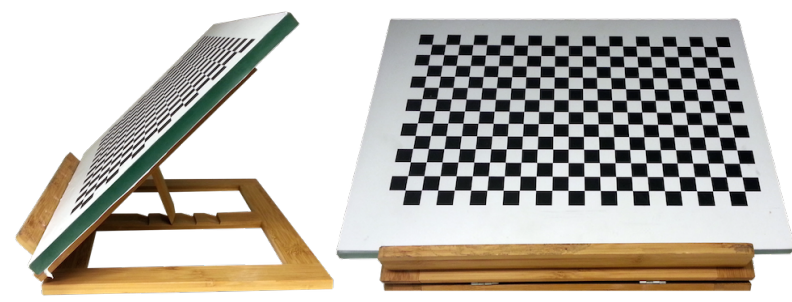

Figure 2: The calibration plate used in this study sitting on a rigid wooden support frame. Manufactured from $(400 \times 280 \times 12 \mathrm{~mm}$ unhardened float-glass. A high resolution printed checkerboard is glued on the flat surface.

Limited work has been conducted on SL parameter investigations and their effect on overall performance (19). However, to the authors knowledge, no quantitative evaluation has been performed on how the different SL parameters directly influence the final results as defined by the VDI/VDE guideline.

\section{EXPERIMENTAL SETUP}

Our structured light setup, as seen in Figure 1, consists of two industrial cameras (Point Grey Research GS3-U3-91S6C-C) and a high resolution DLP projector (LG PF80G) mounted on a rigid aluminum beam structure. Technical specifications are given in Table 1 . In addition, a high precision turntable is used in order to provide automatic rotation of a calibration checkerboard. The apparatus and scan objects can be fully enclosed during capture, in order to prevent ambient light contamination.

\begin{tabular}{|l|l|}
\hline Parameter & Specification \\
\hline \hline Cameras CCD & Sony ICX814 1" \\
Cameras Resolution & $3376 \times 2704 \mathrm{px}$ \\
Camera Lens Focal Length & $16 \mathrm{~mm}$ \\
Camera Lens Aperture & 5.6 \\
Camera Exposure & $66.66 \mathrm{~ms}$ \\
Projector Resolution & $1920 \times 1080 \mathrm{px}$ \\
Camera Baseline & $450 \mathrm{~mm}$ \\
Camera Object Distance & $\sim 750 \mathrm{~mm}$ \\
Stereo Field of View (FOV) & $(300 \times 300 \times 230) \mathrm{mm}$ \\
\hline
\end{tabular}

Table 1: Technical specifications of our structured light setup.

Figure 2 shows the calibration plate and Figure 3 shows the VDI/VDE 2634(2) measurement artifacts used during this study. The arti- facts consist of a flat white painted aluminum plate and two ceramic spheres separated by a known distance. Both artifacts have been measured according to procedure T3-01 of ISM3D using a coordinate measurement machine (CMM), and traceability has been established through the virtual CMM method. Specifications for nominal values and attached uncertainties are listed in Table 2 and 3.

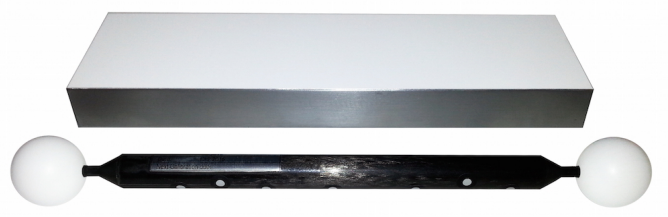

Figure 3: Calibration artifacts according to the VDI/VDE 2634(2) standard. Top: painted and lapped aluminum flat. Bottom: alumina-circonium ceramic spheres on a carbon-fiber rod. Nominal values are given in table 2 and 3.

Following the VDI/VDE 2634 (2), we use four quality parameters:

- Probing error form, $\mathrm{P}_{F}$, which describes the radial range of residuals from a least squares fit sphere with up to $0.3 \%$ of the worst points rejected.

- Probing error shape, $\mathrm{P}_{S}$, measuring the signed deviation between the least squares fit diameter and the nominal. Again, up to $0.3 \%$ of the worst points are rejected.

- Sphere distance error, SD, denoting the signed difference between the estimated and nominal distance between the spheres. Up to $0.3 \%$ of the worst points are rejected.

- Flatness, F, which is the range of residuals from the measured points to a least squares fitted plane, with up to $0.3 \%$ of the worst points rejected.

$\mathrm{P}_{F}$ and $\mathrm{P}_{S}$ are measured using one of the spheres at 10 positions within the system's FOV. SD is measured with the ball-bar at 7 positions, while $\mathrm{F}$ is determined using the flat in 6 positions. These positions are illustrated in Figure 4.

\begin{tabular}{|l|c|}
\hline Parameter & Value \\
\hline \hline Center distance & $198.9612 \mathrm{~mm}$ \\
Distance uncertainty & $0.001 \mathrm{~mm}$ \\
Diameter ball 1 & $24.9989 \mathrm{~mm}$ \\
Diameter ball 2 & $24.9969 \mathrm{~mm}$ \\
Min. dev. from sphere 1 & $-0.0013 \mathrm{~mm}$ \\
Max. dev. from sphere 1 & $+0.0006 \mathrm{~mm}$ \\
Min. dev. from sphere 2 & $-0.0011 \mathrm{~mm}$ \\
Max. dev. from sphere 2 & $+0.0020 \mathrm{~mm}$ \\
Deviation uncertainty & $0.0018 \mathrm{~mm}$ \\
\hline
\end{tabular}

Table 2: Specification of the dumbbell used for our experiments.

\begin{tabular}{|l|c|}
\hline Parameter & Value \\
\hline \hline Minimum deviation from plane & $-0.0030 \mathrm{~mm}$ \\
Maximum deviation from plane & $+0.0012 \mathrm{~mm}$ \\
Deviation uncertainty & $0.0018 \mathrm{~mm}$ \\
\hline
\end{tabular}

Table 3: Specification of the flat plane used for our experiments. 

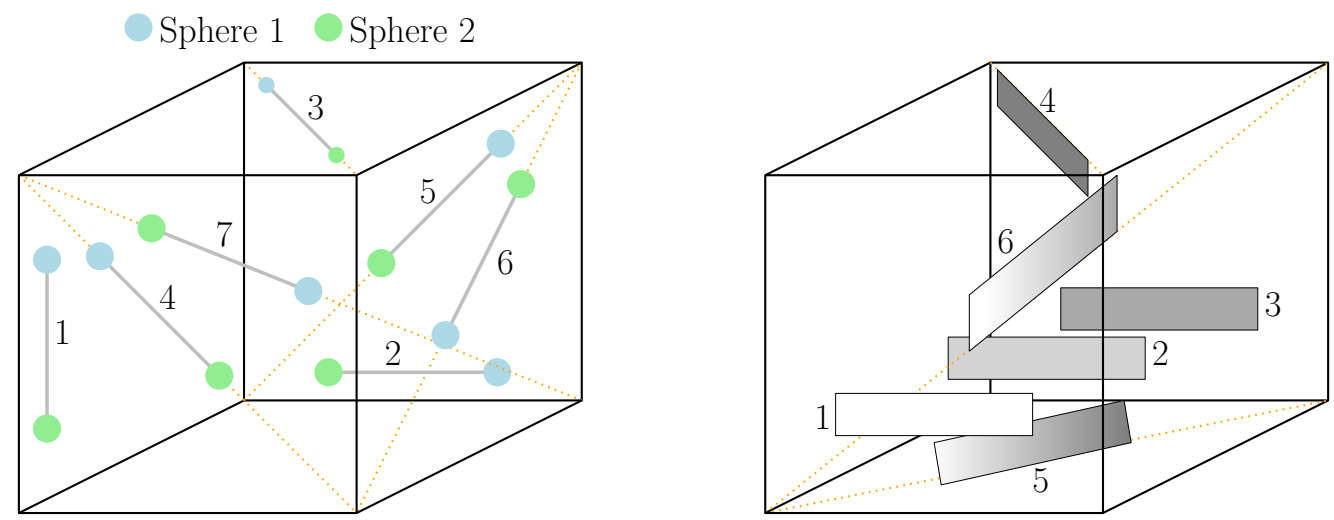

Figure 4: Measurement positions used throughout the paper. The outer frame represents the FOV, as seen from the cameras (Position 1 being closest). Left: ball-bar positions used for sphere distance SD. Right: positions of the flat used for the flatness error metric, F.

\section{CALIBRATION PARAMETERS}

The industry standard models that are essential for calibration of an SL system contain several parameters. Which of these parameters to include in the calibration process is unclear. To solve for the calibration parameters we employ the commonly used method proposed by Zhang (33). We use the 4 parameter pinhole model with the addition of up to five lens distortion parameters. Hence, the camera is modeled as

$$
\left[\begin{array}{ccc}
f_{x} & 0 & c_{x} \\
0 & f_{y} & c_{y} \\
0 & 0 & 1
\end{array}\right]
$$

The use of a non-unit aspect ratio (i.e., $f_{x} \neq f_{y}$ ), makes it possible to model non-square pixels and/or capture compound nonuniformity in the lens. Likewise estimation of the principle point, $\left(c_{x}, c_{y}\right)$, makes it possible to describe cameras in which the principle ray does not strike the image sensor in it's exact center. With quality components such as ours, we would expect these parameters to be unnecessary. At the same time, the inclusion of these parameters increases the risk of false estimation, numerical instability and non-convergence. In fact, it was shown, that principle point estimation is especially prone to misinterpretation, and that the parameter can often be neglected in cameras of medium to long focal length (25).

Radial lens distortion is modeled according to

$$
\begin{aligned}
& x^{\prime}=x\left(1+k_{1} r^{2}+k_{2} r^{4}+k_{3} r^{6}\right) \\
& y^{\prime}=y\left(1+k_{1} r^{2}+k_{2} r^{4}+k_{3} r^{6}\right),
\end{aligned}
$$

where $\left(k_{1}, k_{2}, k_{3}\right)$ are the three distortion coefficients. Tangential distortion is modeled

$$
\begin{aligned}
& x^{\prime}=x+\left(2 p_{1} x y+p_{2}\left(r^{2}+2 x^{2}\right)\right) \\
& y^{\prime}=y+\left(2 p_{1}\left(r^{2}+2 y^{2}\right)+2 p_{2} x y\right)
\end{aligned}
$$

where $\left(p_{1}, p_{2}\right)$ are the tangential distortion parameters. This five parameter "Brown-Conrady" model is widely accepted (5).

The stereo relationship between cameras is described using three rotations and three translations. Due to weak inter-dependencies, the calibration can be performed individually per camera, followed by stereo calibration. Still, the risk of over-fitting and converging to local minima remains, and therefore higher order distortion parameters are used only when considered relevant. To investigate these factors, we calibrate using 8 different configurations of parameters and evaluate by means of VDI/VDE quality parameters. Each calibration is performed using 81 observations of the calibration board, evenly sampled in the range from -40 to 40 degrees relative to baseline.

Figure 5 shows performance results for the different calibration parameter configurations. The baseline setting generally yields sub-millimeter results. The free aspect ratio $\left(f_{x} \neq f_{y}\right)$ and principal point estimation degrade the performance from "baseline". These results show that in a typical setup, omitting the principle point estimation makes calibration significantly more stable. It can be seen that by enabling the first two distortion coefficients, significant improvement is obtained. This is especially noticeable in the sphere distance metric, SD, being a measure of accuracy. No significant improvement is obtained with additional distortion parameters.

Conclusion Given our setup, only the $k_{1}$ and $k_{2}$ distortion coefficients are required for accurate calibration. The inclusion of both aspect ratio and principle point estimation makes the calibration procedure unstable, and considerably better results are obtained without them. With their removal, we see consistently low results of $\mathrm{P}_{F}, \mathrm{SD}$ and $\mathrm{F}$, while the estimation of sphere sizes $\left(\mathrm{P}_{S}\right)$ is biased to positive values. This indicates that one should carefully consider which camera model is used.

\section{CALIBRATION OBSERVATIONS}

An important question in calibration is in which poses the calibration board needs to be observed. Viewing the calibration board at very shallow angles means higher uncertainty in point localization. In addition, the effect of non-planarity becomes larger. However, it is necessary to observe some degree of foreshortening for focal length estimation (33).

In this section we attempt to obtain the optimal angular range of observations relative to the baseline. We tested 8 different ranges starting from $-5^{\circ}$ to $5^{\circ}$ relative to baseline and ending in $-40^{\circ}$ to $40^{\circ}$. For each range, we sample evenly 11 images of the calibration board. For the rotations performed, most foreshortening will be observed around the rotation axis, thus constraining the focal length parameter $f_{x}$ well. With a fixed aspect ratio, this in turn constraints $f_{y}$ also.

The results from the experiment can be seen in Figure 6. It is seen that increased foreshortening affects the sphere distance parameter (SD) positively indicating better calibration. In general, the results are quite comparable for all ranges. Comparing to Figure 5 it is also apparent that using 11 observations and 81 observations ranging from -40 to 40 yields similar results. 

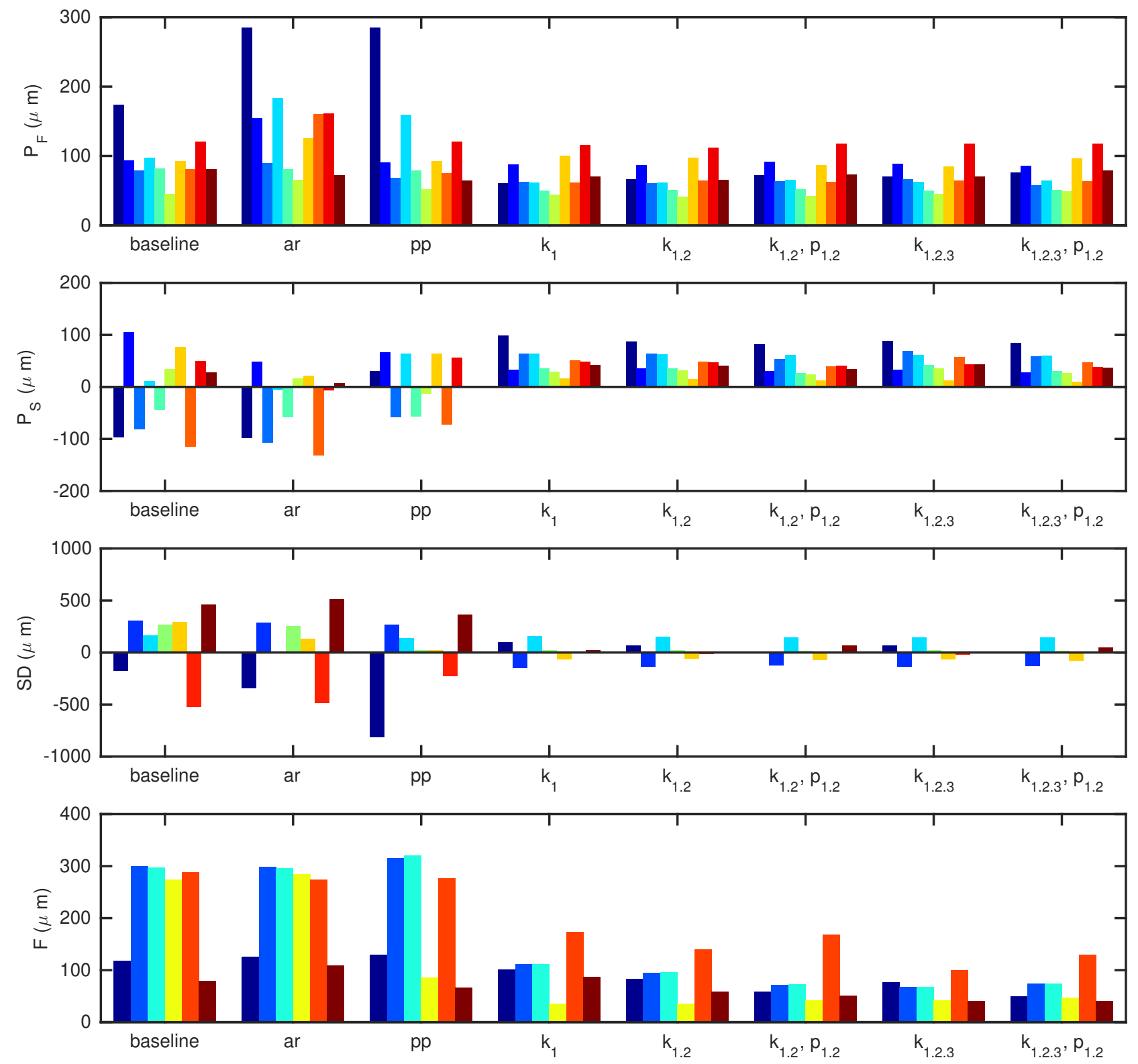

Figure 5: Results obtained with different camera and lens models. Colors represent different positions of the dumbbell or flat artifact according to Fig. 4 (Position 1 being the leftmost bar). Baseline denotes the pinhole model with fixed aspect ratio, fixed principle point and without distortion parameters. "ar" adds aspect aspect ratio, "pp" principle point determination. The other groups show results when different combinations of lens parameters are used. From this we see significant improvements when lens distortion parameters are added. The inclusion of both aspect ratio and principle point estimation makes the calibration procedure unstable, and considerably better results are obtained without them. 
Conclusion In terms of accuracy, it is slightly beneficial to use a large angular range during calibration. However, even a smaller amount of foreshortening is sufficient to accurately estimate parameters. We opt for the $80^{\circ}$ range. Furthermore, the difference between 11 and 81 observations is negligible, thus for the sake of practicality we proceed by using the former.

\section{ENCODING STRATEGIES}

The encoding strategy of a structured light system determines how correspondences are found, and can be expected to be a major factor in system precision. We identify three main categories of algorithms which are relevant in this setting:

- Fourier methods, prominently phase shifting (PS) methods (28).

- Binary boundary based methods (23), e.g. Binary and Gray coding.

- Line shifting (12), which is the same principle underlying triangulation based laser line scanners, with multiple lines sweeping the scene simultaneously.

Phase Shift (PS) based methods encode the scene by means of a series of shifted sinusoidal patterns. The phase is then recovered and matched between cameras (16). The advantage is that the scene can be densely encoded using at least 3 patterns, and more can be naturally added to increase precision. For correct encoding, the projector-camera system should have a close to linear intensity-response. The frequency of sinusoids can also be altered, with higher frequencies yielding higher precision at the cost of phase ambiguities, which then have to be "unwrapped" using additional patterns. Our PS implementation performs 32 steps at the highest frequency sinusoidal pattern (period $19.2 \mathrm{px}$ ), and unwraps the resulting phase using two sets of lower frequency patterns (34). The total number of projected patterns is 64 .

Binary boundary based methods, such as the Gray Code method, encode scene points directly by means of binary codes, which are decoded and matched in the cameras. These methods are flexible in the number of patterns, and allow for the natural addition of redundant information, which can then be used to reject outliers. Feature points locations can be estimated with sub-pixel accuracy. Our Gray code implementation encodes uniquely every other column in projector space, and employs patterns and their inverse for added robustness. Boundaries are detected at the intersection of the pattern and its inverse with subpixel accuracy. The total number of patterns is 20 .

Line shifting can be performed with a single laser line as the projection source, however with a digital projector, many lines can be projected in parallel. Correspondence points are found at the peak of the stripes. Several methods exist for subpixel peak detection (29). For Gühring's Line Shifting method, we employ Gray codes to partition the encoded space into 256 unique regions. For each of these regions, a single projector line then walks across it in 8 steps, resulting in a total of 28 patterns. The peak of each single line is determined as the first derivative zero crossing using a Gaussian derivative filter of size $5 \mathrm{px}$.

These classes of encoding strategies have fundamentally different error characteristics. The binary and line shifting methods may be very robust against point outliers, but PS patterns are often less affected by projector and camera blur due to their low-frequency nature.

\begin{tabular}{|l|c|c|c|}
\hline Parameter & PS & Gray & Line Shift \\
\hline \hline Number of patterns & 64 & 20 & 28 \\
Off-focus robustness & Excellent & Good & Moderate \\
Precision & Excellent & Good & Good \\
Accuracy & Excellent & Excellent & Excellent \\
Nr. of points (sphere) & $\sim 20 \mathrm{k}$ & $\sim 7 \mathrm{k}$ & $\sim 10 \mathrm{k}$ \\
Nr. of points (flat) & $\sim 450 \mathrm{k}$ & $\sim 150 \mathrm{k}$ & $\sim 250 \mathrm{k}$ \\
\hline
\end{tabular}

Table 4: Interpretation of the algorithm performance.

The SL system is calibrated with the previously determined angular range of 80 degrees and 11 positions. Furthermore, we use the $k_{1,2}$ parameter selection, as previously identified. A comparison of the VDI/VDE quality parameter results for these three encoding strategies is seen in Figure 7. A summary of the results may be seen in Table 4 .

The precision of the different strategies can be indirectly estimated from the spherical form parameter $P_{F}$. This is because the calibration spheres cover only a small part of the scanning volume, whereas the flat plane occupies a substantial part. Flat plane measurements will thus be more affected by the quality of calibration and lens distortion correction. Both of which directly affect precision and accuracy.

From the results we confirm that the PS method is more tolerant to depth of field limitations, where positions close and far away show no signs of degradation. The PS method also shows superior precision characteristics in the $P_{F}$ parameter. For the $P_{S}$ parameter, there is a clear bias present in both the PS and Gray code method, whereas Line Shift appears bias free. Figure 8 illustrates sphere fitting results for the three methods. Here it is seen, that PS and Gray have systematic positive residuals towards the lateral edge in horizontal (encoding) direction. This in turn leads to slight overestimation of sphere diameters.

Conclusion In the quality parameters $\mathrm{P}_{F}$ (Spherical form) and $\mathrm{F}$ (Flatness), the PS encoding strategy provides the best results in all artifact positions. The sphere diameter is biased positively in the PS and Gray methods, while it appears unbiased with Line Shift. Overall it appears that the PS method is the best performing method.

\section{COMPARISON TO METROLOGY SCANNER}

Finally, we have compared our system to a high-end commercial scanner (GOM ATOS III Triple Scan), which has a FOV of $240 \times$ $320 \times 240 \mathrm{~mm}$, similar to the FOV of our system (see Table 1).

In this experiment we used the PS algorithm, and calibrated as in the preceding experiment. Again, quality measures defined by the VDI/VDE 2634(2) were measured. Results are seen in Figure 9. The results show that our system is more precise and in terms of $\mathrm{P}_{F}$ and exhibits lower variance. However, a bias is present in the $\mathrm{P}_{S}$ whereas the commercial system appears free of such. It is apparent from the sphere spacing term (SD) that the commercial system indicates better accuracy.

Interestingly, the GOM scanner shows significant improvements in the flatness form error metric, F, compared to the sphere form, $\mathrm{P}_{F}$, where one would expect similar performance (as seen in our system). Reasons for this will only be cause for speculation as the scanning procedure and reconstruction is proprietary, that being said, some form of smoothing favoring planar surfaces might be at work.

Conclusion Our system generally performs better in the precision characteristic, $\mathrm{P}_{F}$, while the the metrology scanner obtains 

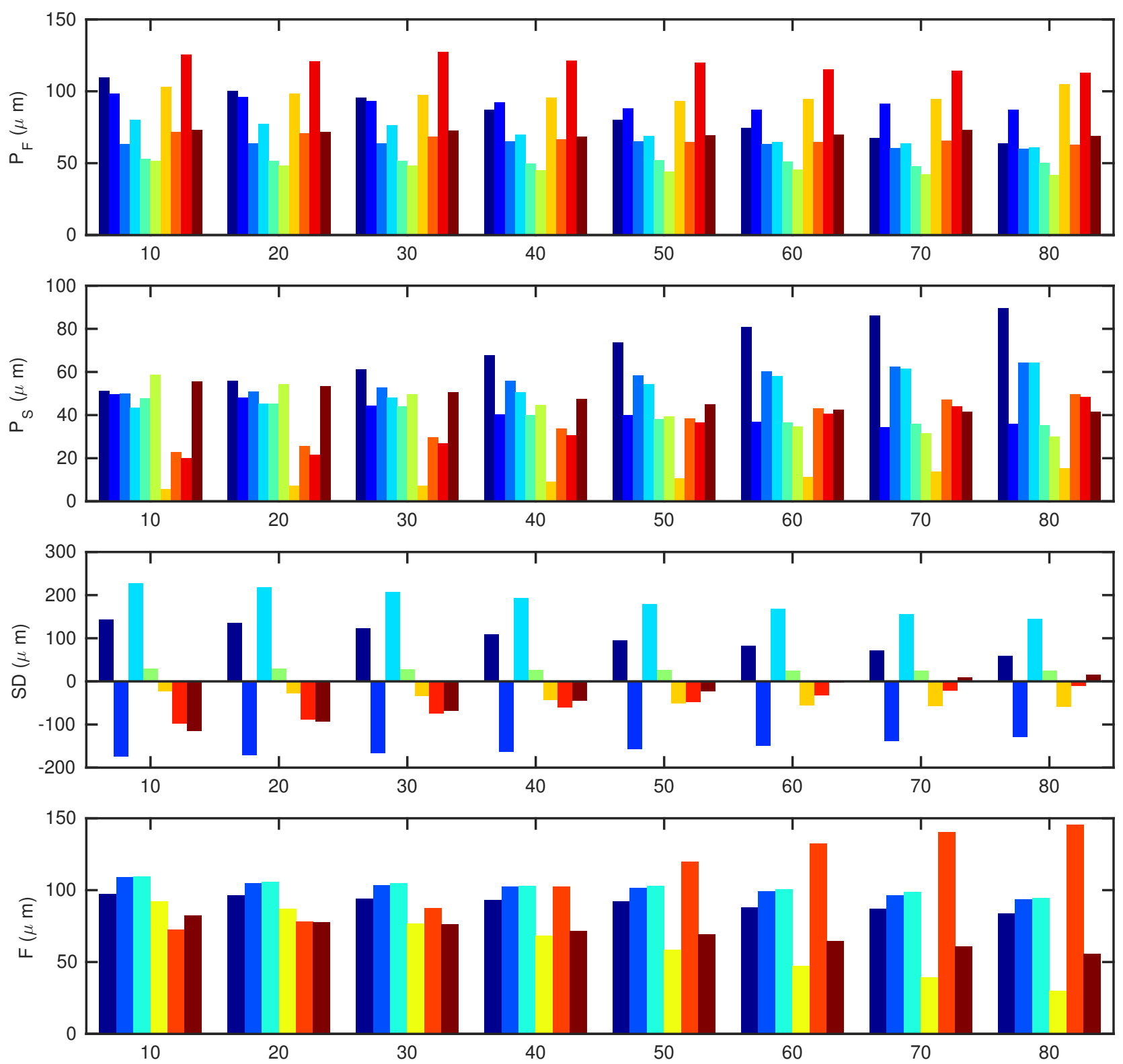

Figure 6: Results obtained with different angular ranges of the calibration plate relative to the camera baseline. Colors represent different positions of the artifacts according to Fig. 4 (Position 1 being the leftmost bar). We see that in terms of accuracy, it is slightly beneficial to use a large angular range during calibration. However, even a smaller amount of foreshortening is sufficient to accurately estimate parameters. 

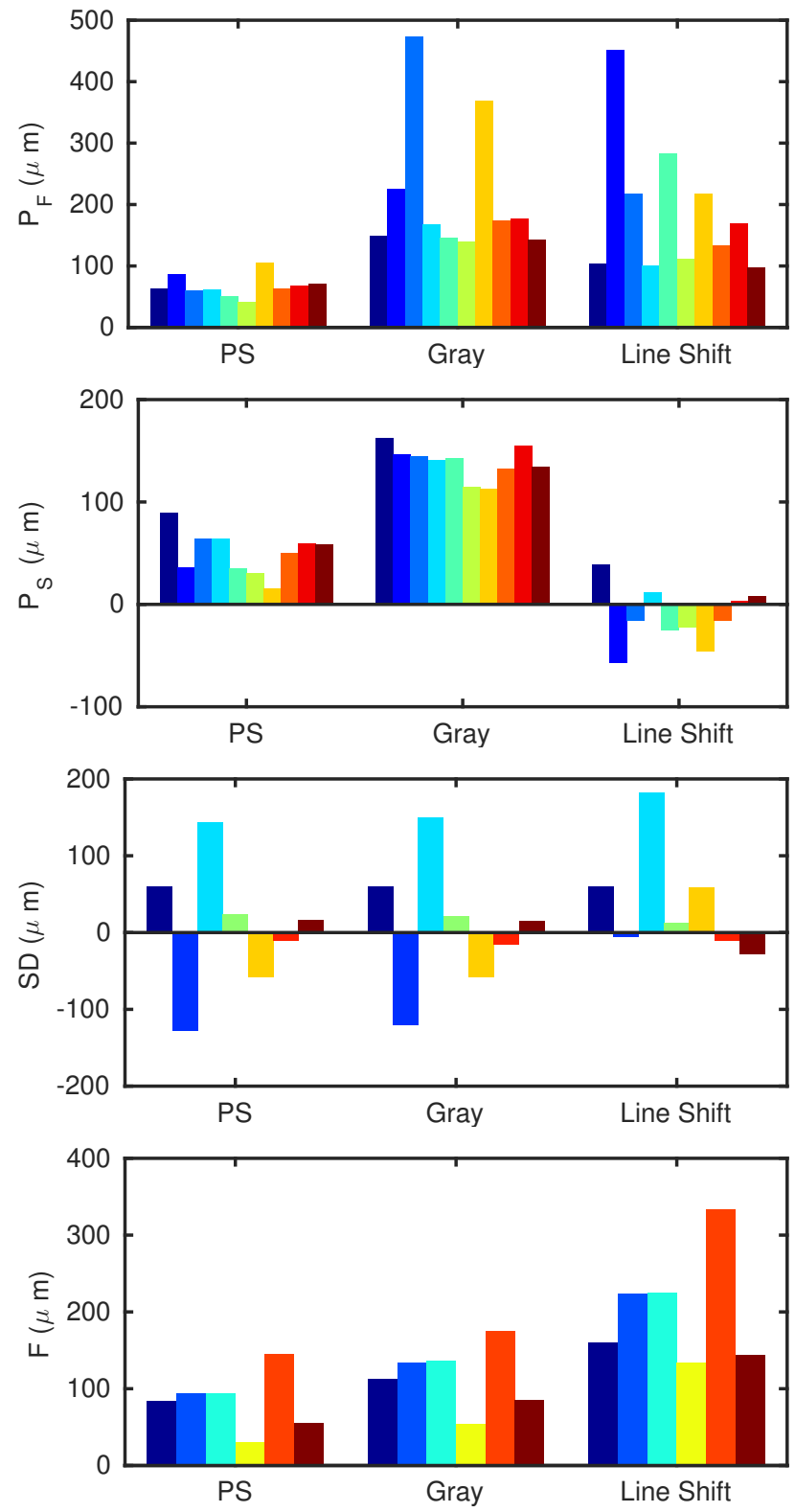

Figure 7: Results obtained with different encoding strategies. Colors represent different positions of the ball-bar or flat artifact according to Fig. 4 (Position 1 being the leftmost bar). Based on this we conclude the PS method to be the overall best performer, yielding significantly higher precision.

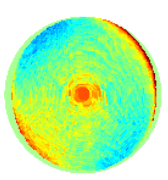

PS

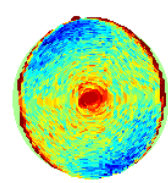

Gray

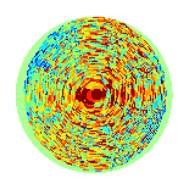

Line Shift

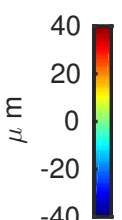

$-40$

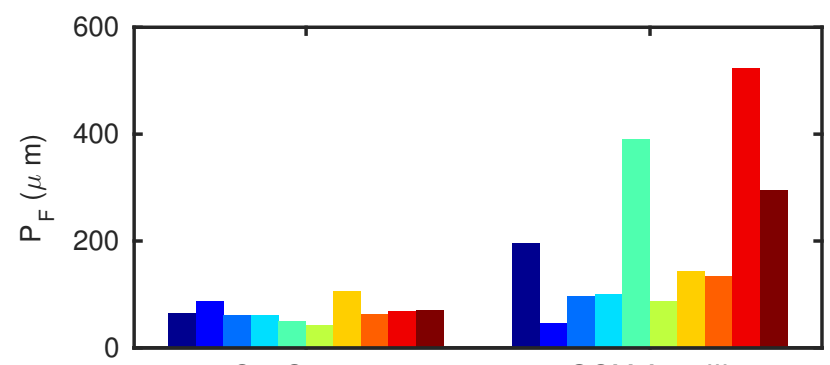

Our System

GOM Atos III
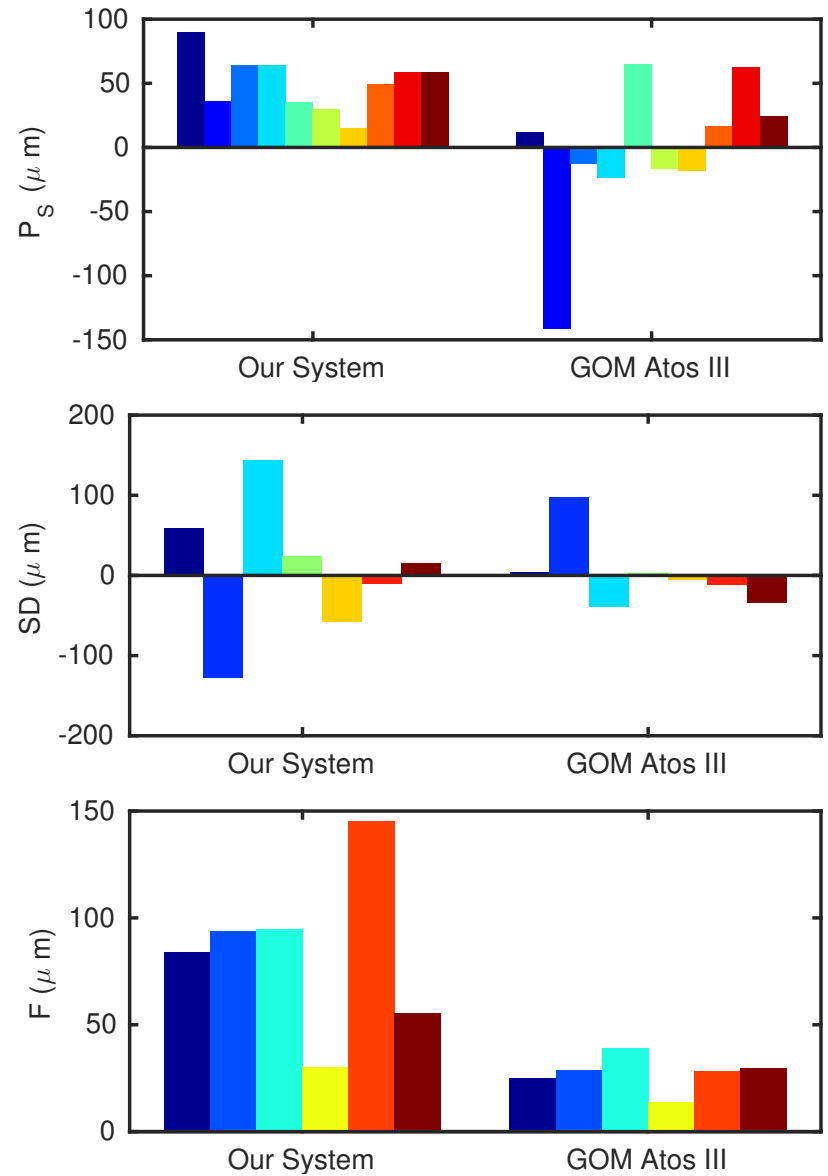

Figure 9: Results obtained with our system compared to results from a commercial metrology grade structured light scanner (GOM Atos III). Colors represent different positions of the dumbbell or flat artifact according to Fig. 4 (Position 1 being the leftmost bar).

Figure 8: Fitted spheres in position 1 for our three algorithms. In PS and Gray, a bias is observed toward the edges in the horizontal (pattern encoding/epipolar line) direction, while Line Shift does not suffer from this bias. This results in an over estimation of the diameter, $\mathrm{P}_{S}$, as seen in Figure 7. 
unbiased sphere diameter results and achieves higher accuracy. Since accuracy is a deterministic noise component, this indicates that a custom calibration method could be advantageous.

\section{DISCUSSION AND CONCLUSION}

In conclusion, in this paper we have shown through quantitative analysis how the most common parameters within structured light systems affect the overall performance. Our quantitative measure is the accepted VDI/VDE 2634(2) guideline which nicely captures critical parameters in terms of precision and accuracy. We perform a series of experiments on our experimental setup using precision made calibration artifacts. We start by investigating calibration parameters as defined by the most commonly used models and follow by determining the angular foreshortening and the amount of observations required to yield the best results. We proceed by comparing three commonly used algorithms against each other in order to determine the best method. Finally, we compare our setup to a metrology grade commercial scanner, using the previously determined parameters. Our results show that comparable and in some cases better results can be obtained using standard methods and models if care is taken in the parameters choice. We expect these findings to be of help to practitioners wanting to build their own SL systems.

Even though the VDI guideline indirectly captures some of the error sources, such as depth of field, calibration performance and acquisition noise, it is lacking to some extent. The calibration shapes suggested consists of low frequency features, thus a Gaussian filtering operation on the measured point cloud will yield better results for some of the parameters. Although it is stated that all filtering operations must be noted; In many cases these filtering operations are required or inherent in the triangulation algorithms at a pre point cloud level. The use of such filtering will affect the frequency response of the system, where low-pass operations will limit the systems capability of resolving high frequency features. In order to better analyze a systems performance, a frequency analysis must be conducted, indicating if any such smoothing is taking place. Such a frequency response characterization calls for an additional calibration artifact in the form of a high frequency feature. In addition, results from the VDI/VDE only provide quantitative evaluation of the artifacts used. Thus the results cannot be transferred to other less optically ideal materials.

\section{REFERENCES}

The Association of German Engineers. VDI-Standard: VDI/VDE 2634 Optical 3-D measuring systems - Optical systems based on area scanning. Beuth Verlag, 2012.

J.-A. Beraldin, F. Blais, S. El-Hakim, L. Cournoyer, and M. Picard. Traceable $3 \mathrm{~d}$ imaging metrology: Evaluation of $3 \mathrm{~d}$ digitizing techniques in a dedicated metrology laboratory. Proc. 8 th Conf. on Optical 3-D Measurement Techniques, 2007.

J.-A. Beraldin and M. Gaiani. Evaluating the performance of close-range $3 \mathrm{~d}$ active vision systems for industrial design applications. Proc. SPIE 5665, Videometrics VIII, 2005.

J. Boehm. Accuracy investigation for structured-light based consumer 3d sensors. Photogrammetrie, Fernerkundung, Geoinformation, 2014(2):117-127, 2014.

D. C. Brown. Decentering distortion of lenses. Photometric Engineering, 32(3):444-462, 1966.

S. Chen, Y. F. Li, and J. Zhang. Vision processing for realtime 3-d data acquisition based on coded structured light. IEEE Trans. Image Processing, 17(2):167-176, 2008.
R. B. Fisher, A. Ashbrook, C. Robertson, and N. Werghi. A lowcost range finder using a visually located, structured light source. Proc. Int. Conf. on 3-D Digital Imaging and Modeling 1999, 24 33, 1999.

J. Geng. Structured-light 3d surface imaging: a tutorial. Advances in Optics and Photonics, 3(2):128-160, 2011.

A. Georgopoulos, C. Ioannidis, and A. Valanis. Assessing the performance of a structured light scanner. Int. Archives of Photogrammetry, Remote Sensing and Spatial Information Sciences, 38, 2010.

S. S. Gorthi and P. Rastogi. Fringe projection techniques: whither we are? Optics and lasers in engineering, 48(2009-001):133$140,2010$.

J. Gühring. Dense 3d surface acquisition by structured light using off-the-shelf components. SPIE Photonics West 2001, 220-231, 2000 .

J. Gühring. Dense 3D surface acquisition by structured light using off-the-shelf components. SPIE Photonics West 2001, 220231, 2000.

G. Guidi. Metrological characterization of $3 \mathrm{~d}$ imaging devices. SPIE Optical Metrology 2013, 2013.

J. Heikkila and O. Silvén. A four-step camera calibration procedure with implicit image correction. Proc. CVPR, 1106-1112, 1997.

L. Iuliano, P. Minetola, and A. Salmi. Proposal of an innovative benchmark for comparison of the performance of contactless digitizers. Measurement Science and Technology, 21(10):105102, 2010.

P. Kühmstedt, C. Munckelt, M. Heinze, C. Bräuer-Burchardt, and G. Notni. 3d shape measurement with phase correlation based fringe projection. SPIE Optical Metrology, 66160B-66160B, 2007.

R. Legarda-Sa, T. Bothe, W. P. Ju, et al. Accurate procedure for the calibration of a structured light system. Optical Engineering, 43(2):464-471, 2004.

Z. Li, Y. Shi, C. Wang, and Y. Wang. Accurate calibration method for a structured light system. Optical Engineering, 47(5):053604-053604, 2008.

W. Lohry, Y. Xu, and S. Zhang, Optimal checkerboard selection for structured light system calibration. SPIE Optics + Photonics, 743202-743202, 2009.

T. Luhmann. 3d imaging: how to achieve highest accuracy. SPIE Optical Metrology, 808502-808502, 2011.

D. MacKinnon, B. Carrier, J.-A. Beraldin, and L. Cournoyer. Gd\&t-based characterization of short-range non-contact 3d imaging systems. Int. Journal of Computer Vision, 102(1-3):56-72, 2013.

B. Møller, I. Balslev, and N. Krüger. An automatic evaluation procedure for $3 \mathrm{~d}$ scanners in robotics applications. IEEE Sensors Journal, 13(2):870-878, 2013.

J. Posdamer and M. Altschuler. Surface measurement by spaceencoded projected beam systems. Computer Graphics and Image Processing, 18(1):1-17 1982.

C. Rocchini, P. Cignoni, C. Montani, P. Pingi, and R. Scopigno. A low cost $3 \mathrm{~d}$ scanner based on structured light. Computer Graphics Forum, 20:299-308, 2001.

A. Ruiz, P. E. López-de Teruel, and G. García-Mateos. A note on principal point estimability. Proc. Pattern Recognition, 2:304307, 2002.

J. Salvi, J. Pages, and J. Batlle. Pattern codification strategies in structured light systems. Pattern Recognition, 37(4):827-849, 2004. 
G. Sansoni, M. Carocci, and R. Rodella. Three-dimensional vision based on a combination of gray-code and phase-shift light projection: analysis and compensation of the systematic errors. Applied Optics, 38(31):6565-6573, 1999.

V. Srinivasan, H.-C. Liu, and M. Halioua. Automated phasemeasuring profilometry: a phase mapping approach. Applied Optics, 24(2):185-188, 1985.

E. Trucco, R. B. Fisher, A. W. Fitzgibbon, and D. Naidu. Calibration, data consistency and model acquisition with laser stripers. Int. Journal of Computer Integrated Manufacturing, 11(4):293310, 1998

M.-J. Tsai and C.-C. Hung. Development of a high-precision surface metrology system using structured light projection. Measurement, 38(3):236-247, 2005.

R. Y. Tsai. A versatile camera calibration technique for highaccuracy $3 \mathrm{~d}$ machine vision metrology using off-the-shelf tv cameras and lenses. IEEE Journal of Robotics and Automation, 3(4):323-344, 1987.

S. Zhang and P. S. Huang. Novel method for structured light system calibration. Optical Engineering, 45(8):083601-083601, 2006.

Z. Zhang. A flexible new technique for camera calibration. IEEE Trans. PAMI, 22(11):1330-1334, 2000.

R. Zumbrunn. Automated fast shape determination of diffuse reflecting objects at close range, by means of structured light and digital phase measurement. In ISPRS Intercommission Conference on Fast Processing of Photogrametric Data, 363-379, 1987. 